\title{
ALTERNATIVAS DE ROTAS PARA BENEFICIAMENTO DE MINÉRIO DE FERRO*
}

Michelle Lacerda Sales Marques ${ }^{1}$ Klaydison Carlaile Silva ${ }^{2}$ Neymayer Pereira Lima ${ }^{3}$

\section{RESUMO}

A busca de novas rotas de beneficiamento que possam maximizar a recuperação metalúrgica e mássica é uma constante na Vale. O trabalho foi desenvolvido em três fases, onde foram estudadas quatro rotas de beneficiamento que buscaram reduzir a geração de ultrafinos e, assim, aumentar a recuperação mássica. Foram avaliadas rotas com pré-concentração do minério em concentração magnética (rota denominada PCM) e flotação (rota denominada MFMF), e uma rota onde a moagem mais grossa (P80 0,15mm) permite uma menor geração de ultrafinos (rota denominada MDMF). Esta última apresentou um CAPEX 6,5\% menor quando comparado a rota PCM.

Palavras-chave: Moagem; Flotação; Separação magnética.

\section{ALTERNATIVE ROUTES FOR IRON ORE BENEFICIATION}

\section{Abstract}

The search for new beneficiation's routes that maximize mass and metallurgical yield is a routine in Vale. The study was carry out in three steps and was tested four processing routes that had the objective to reduce the generation of ultrafine and thus increase the yield. Routes with pre-concentration of ore were evaluated on magnetic concentration (called PCM route) and flotation (called MFMF route), and a route where with a coarser grind (P80 $0.15 \mathrm{~mm}$ ) allows a smaller generation of ultrafines (called MDMF route). These route presented 6.5\% lower CAPEX compared with PCM route.

Keywords: Grinding; Flotation; Magnetic separation.

1 Engenheira de Minas, Mestre, Vale, Desenvolvimento de Processos de Tratamento de Minério, Nova Lima, MG, Brasil.

2 Engenheiro de Minas, Mestre, Engenheiro Master, Vale, Desenvolvimento de Processos de Tratamento de Minério, Nova Lima, MG, Brasil.

3 Engenheiro de Minas, Doutor, Engenheiro Master, Vale, Desenvolvimento de Processos de Tratamento de Minério, Nova Lima, MG, Brasil.

* Contribuição técnica ao 44 Seminário de Redução de Minério de Ferro e Matérias-primas, $15^{\circ}$ Simpósio Brasileiro de Minério de Ferro e $2^{\circ}$ Simpósio Brasileiro de Aglomeração de Minério de Ferro, 15 a 18 de setembro de 2014, Belo Horizonte, MG, Brasil. 


\section{INTRODUÇÃO}

A busca de novas rotas de beneficiamento que possam maximizar a recuperação metalúrgica e mássica é uma constante na Vale. Além disso, a redução do CAPEX em novos projetos e a necessidade de melhorar a atratividade dos mesmos tem sido os motivadores para o desenvolvimento de novas rotas de tratamento.

A moagem direta na malha de liberação promove alta geração de ultrafinos que são perdidos na etapa de deslamagem, principalmente para minérios com liberação do quartzo em malhas como $0,045 \mathrm{~mm}$. Assim, algumas rotas têm sido avaliadas de forma a mitigar este fator, tendo sido estudadas quatro rotas, a saber:

- Rota MF: consiste na moagem com classificação na malha de liberação seguida de deslamagem e flotação.

- Rota PCM: consiste na moagem e classificação com P80 em torno de $0,15 \mathrm{~mm}$, seguido de uma etapa de separação magnética, moagem na malha de liberação do minério e posterior flotação.

- Rota MFMF: consiste na moagem e classificação com top size em $0,6 \mathrm{~mm}$, deslamagem e posteriormente flotação, sendo o concentrado remoído na malha de liberação e flotado novamente.

- Rota MDMF: consiste na moagem e classificação com P80 em torno de $0,15 \mathrm{~mm}$, seguida de deslamagem e cominuição do underflow na malha de liberação para posterior flotação.

Os estudos foram realizados em diferentes momentos e continuam em andamento, mas produzem subsídios consistentes para tomada de decisão dos novos projetos da empresa.

\section{MATERIAIS E MÉTODOS}

O estudo foi desenvolvido em três fases, sendo na primeira realizado um comparativo entre a rota denominada PCM e a atual rota aplicada no beneficiamento do minério de ferro (rota MF) em escala de laboratório. Foram coletadas amostras de itabirito friável do Quadrilátero Ferrífero e realizados ensaios conforme fluxograma a seguir.

* Contribuição técnica ao $44^{\circ}$ Seminário de Redução de Minério de Ferro e Matérias-primas, $15^{\circ}$ Simpósio Brasileiro de Minério de Ferro e $2^{\circ}$ Simpósio Brasileiro de Aglomeração de Minério de Ferro, 15 a 18 de setembro de 2014, Belo Horizonte, MG, Brasil. 

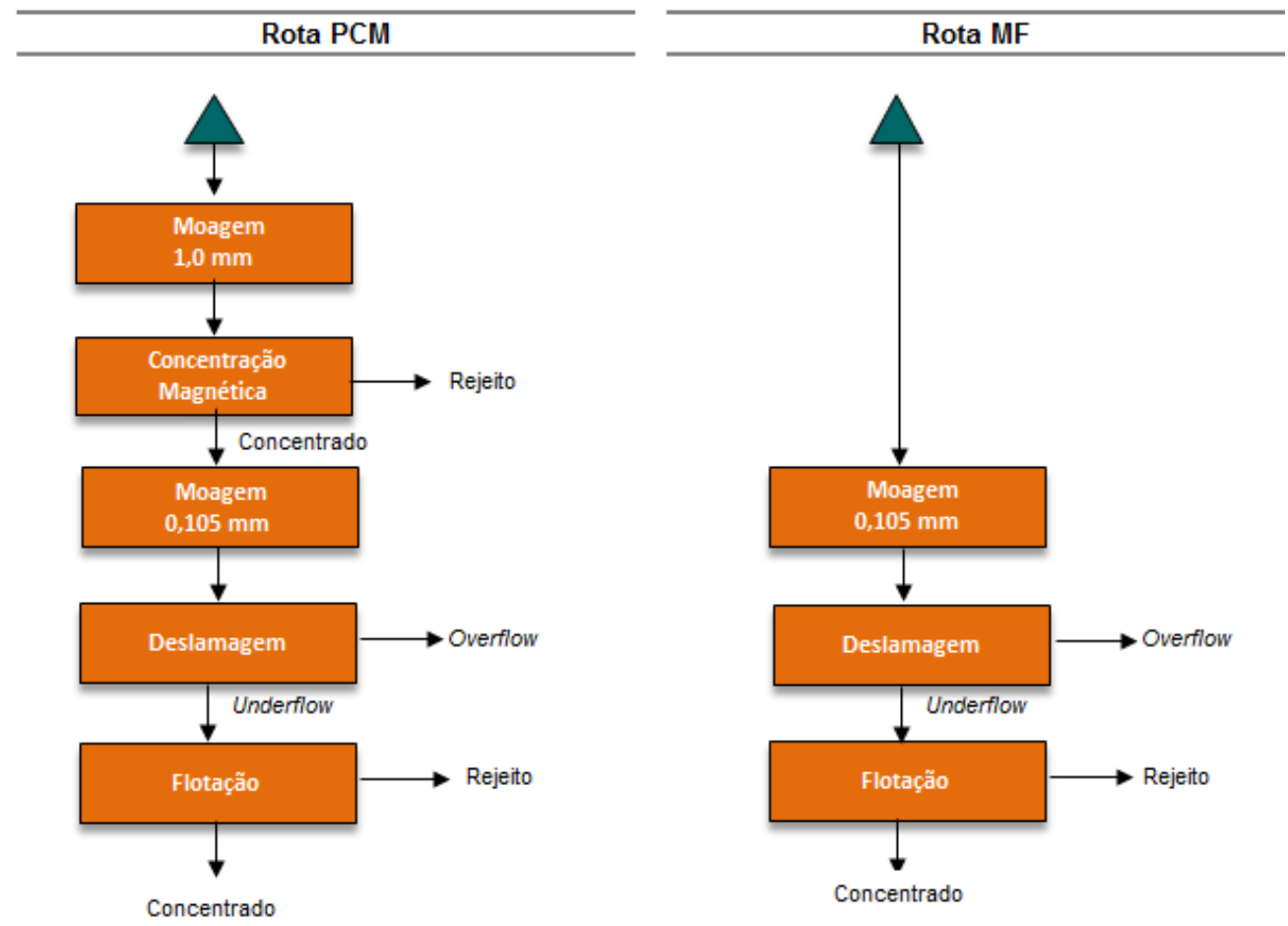

Figura 1 - Fluxograma testes fase 1 - PCM versus MF.

Minérios com malha de liberação inferiores a $0,105 \mathrm{~mm}$ submetidos a rota MF produzem grandes quantidades de ultrafinos. Desta forma, na segunda fase do estudo definiu-se avaliar uma rota mais adequada a este tipo de material como a MFMF, onde a geração de lamas ocorre em malha similar a minérios cuja liberação acontece em malhas mais grossas.

Diante do potencial da rota PCM em escala de laboratório, a mesma foi testada em escala piloto. Para ambas as rotas utilizou-se um blend de itabirito compacto (40\%) e friável $(60 \%)$ cuja liberação é em $0,045 \mathrm{~mm}$. A figura a seguir apresenta o fluxograma das rotas testadas.
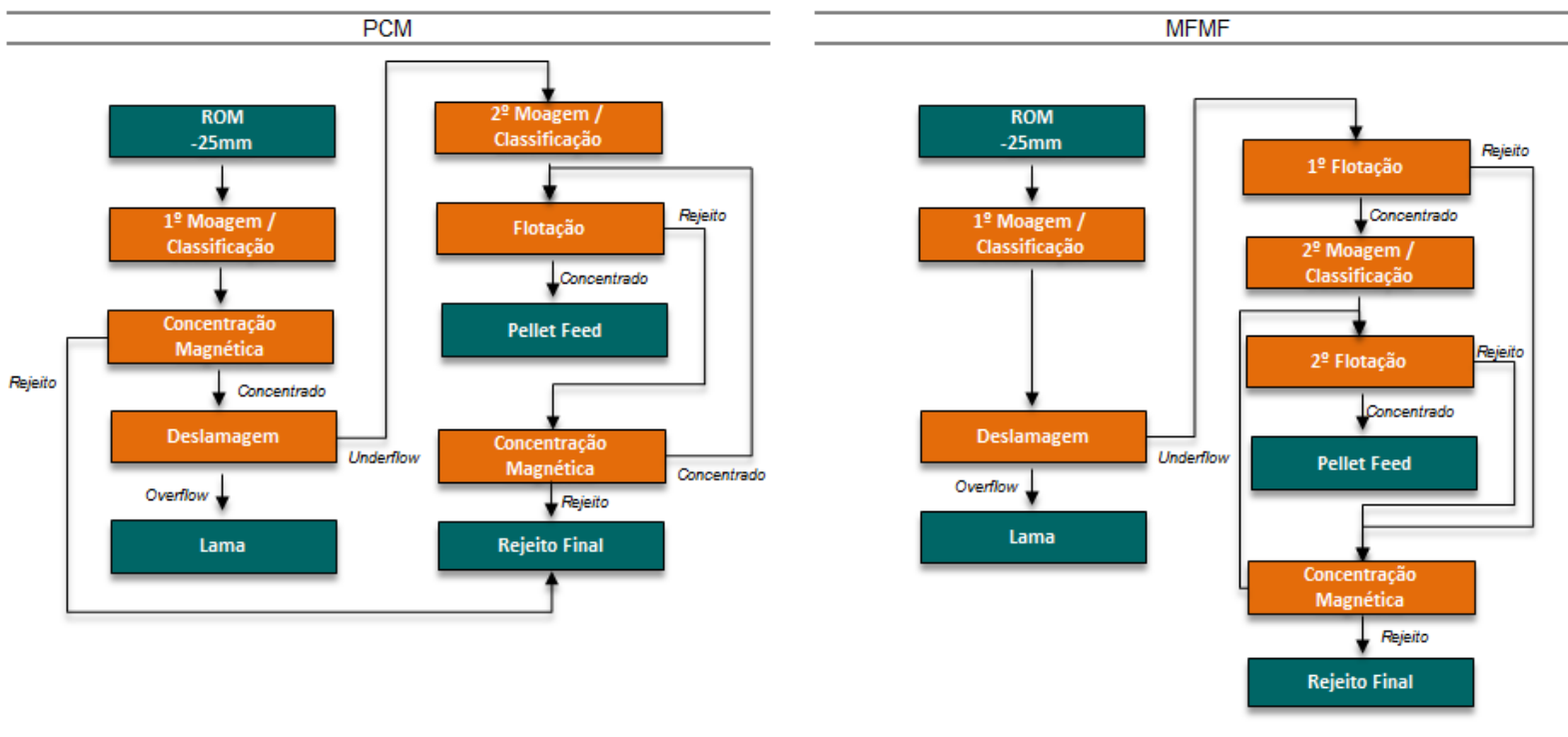

Figura 2 - Fluxograma testes fase 2 - PCM versus MFMF.

* Contribuição técnica ao 44 Seminário de Redução de Minério de Ferro e Matérias-primas, 15오 Simpósio Brasileiro de Minério de Ferro e 2 Simpósio Brasileiro de Aglomeração de Minério de Ferro, 15 a 18 de setembro de 2014, Belo Horizonte, MG, Brasil. 
$\mathrm{Na}$ terceira fase foi desenvolvida uma nova rota denominada MDMF que consiste em cominuir o minério com P80 em torno de $0,15 \mathrm{~mm}$, deslamagem, seguida da cominuição do minério na malha de liberação. Essa rota foi desenvolvida para minérios com malha de liberação superiores a $0,105 \mathrm{~mm}$.

A figura a seguir apresenta o fluxograma da rota.

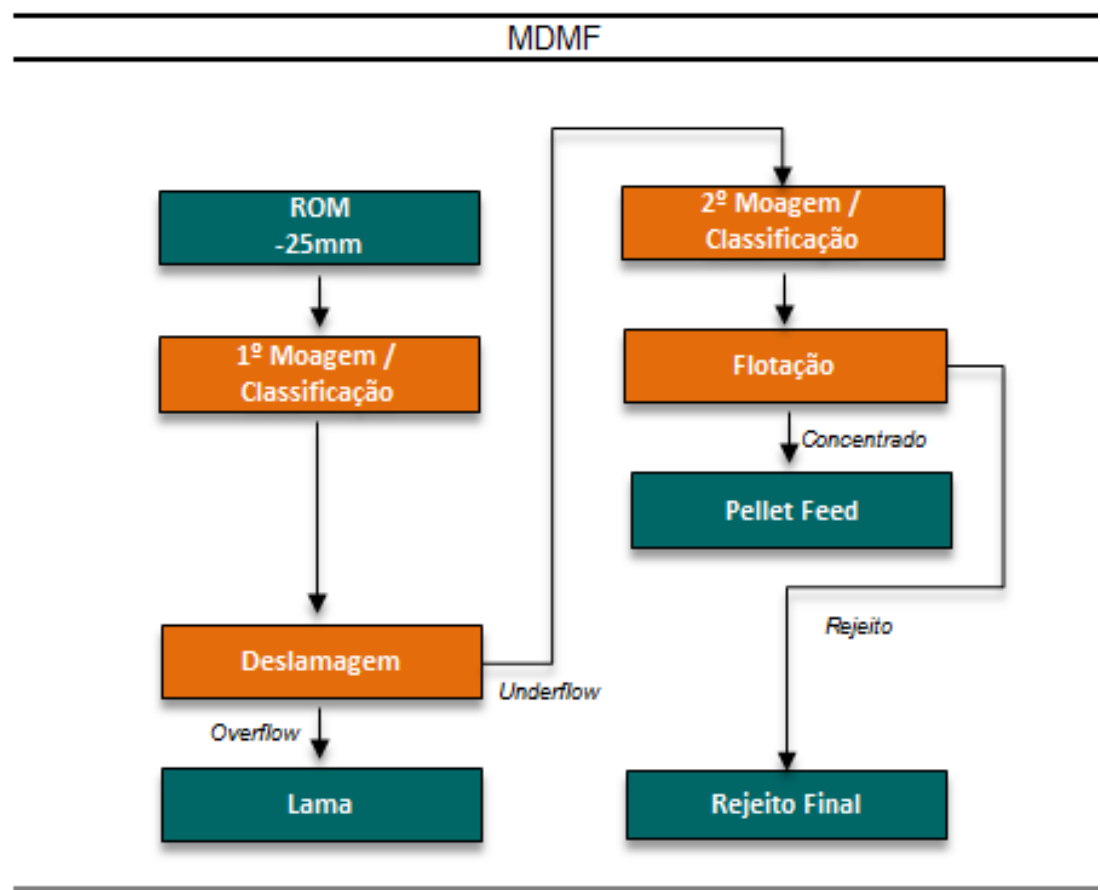

Figura 3 - Fluxograma rota MDMF.

Diante do potencial da rota PCM e baseado nos resultados dos testes piloto da segunda fase, realizou-se uma simulação no software Usimpac a fim de compará-la com a rota MDMF, uma vez que ambas possuem menor geração de ultrafinos. Realizou-se a partir destes balanços de massas o pré-dimensionamento dos equipamentos e estimou-se o CAPEX para cada caso.

\section{RESULTADOS E DISCUSSÃO}

A tabela a seguir apresenta os resultados em escala de laboratório do comparativo entre as rotas PCM e MF. Como não foram realizados ensaios da etapa scavenger, a fim de comparar os resultados, para ambos os circuitos considerou-se um teor final na concentração de $12 \%$ de Fe.

* Contribuição técnica ao 44 Seminário de Redução de Minério de Ferro e Matérias-primas, 15오 Simpósio Brasileiro de Minério de Ferro e 2 Simpósio Brasileiro de Aglomeração de Minério de Ferro, 15 a 18 de setembro de 2014, Belo Horizonte, MG, Brasil. 
Tabela 1 - Resultados 1ํ Fase - Rota PCM versus MF

Rota PCM 0,105mm

\begin{tabular}{|c|c|c|c|c|c|c|c|}
\hline Fluxo & $\begin{array}{c}\% \text { Massa } \\
\text { AM }\end{array}$ & $\begin{array}{c}\% \mathrm{Fe} \\
\mathrm{AM}\end{array}$ & $\begin{array}{c}\% \mathrm{SiO}_{2} \\
\mathrm{AM}\end{array}$ & $\begin{array}{l}\% \mathrm{P} \\
\mathrm{AM}\end{array}$ & $\begin{array}{c}\% \mathrm{Al}_{2} \mathrm{O}_{3} \\
\mathrm{AM}\end{array}$ & $\begin{array}{c}\% \mathrm{Mn} \\
\mathrm{AM}\end{array}$ & $\begin{array}{c}\% \text { PPC } \\
\text { AM }\end{array}$ \\
\hline Alimentação & 100,0 & 46,0 & 32,2 & 0,025 & 0,85 & 0,020 & 0,54 \\
\hline Concentrado Concentração Magnética & 70,5 & 62,1 & 10,0 & 0,029 & 0,50 & 0,042 & 0,47 \\
\hline Rejeito Concentração Magnética & 29,5 & 11,6 & 80,2 & 0,029 & 2,10 & 0,007 & 1,14 \\
\hline Underflow Deslamagem & 60,5 & 62,5 & 9,7 & 0,024 & 0,22 & 0,020 & 0,44 \\
\hline Overflow Deslamagem & 9,9 & 56,1 & 16,0 & 0,070 & 1,69 & 0,036 & 1,60 \\
\hline Pellet Feed & 53,4 & 69,2 & 0,5 & 0,028 & 0,14 & 0,023 & 0,42 \\
\hline Rejeito Flotação & 7,1 & 12,0 & 82,0 & 0,011 & 0,38 & 0,009 & 0,27 \\
\hline
\end{tabular}

Rota MF 0,105mm

\begin{tabular}{|l|c|c|c|c|c|c|c}
\multicolumn{1}{c|}{ Fluxo } & $\begin{array}{c}\text { \% Massa } \\
\text { AM }\end{array}$ & $\begin{array}{c}\% \mathrm{Fe} \\
\mathbf{A M}\end{array}$ & $\begin{array}{c}\% \mathrm{SiO}_{2} \\
\mathbf{A M}\end{array}$ & $\begin{array}{c}\% \mathbf{P} \\
\mathbf{A M}\end{array}$ & $\begin{array}{c}\% \mathrm{Al}_{2} \mathrm{O}_{3} \\
\mathbf{A M}\end{array}$ & $\begin{array}{c}\% \text { Mn } \\
\mathbf{A M}\end{array}$ & $\begin{array}{c}\% \mathrm{PPC} \\
\mathbf{A M}\end{array}$ \\
\hline Alimentação & 100,0 & 46,0 & 32,2 & 0,025 & 0,85 & 0,020 & 0,54 \\
\hline Underflow Deslamagem & 83,8 & 47,0 & 32,0 & 0,020 & 0,32 & 0,012 & 0,23 \\
\hline Overflow Deslamagem & 16,2 & 39,9 & 35,4 & 0,109 & 4,61 & 0,012 & 2,52 \\
\hline Pellet Feed & $\mathbf{5 1 , 5}$ & $\mathbf{6 9 , 0}$ & $\mathbf{0 , 5}$ & $\mathbf{0 , 0 3 0}$ & $\mathbf{0 , 1 6}$ & $\mathbf{0 , 0 1 4}$ & $\mathbf{0 , 4 0}$ \\
\hline Rejeito Flotação & 32,3 & 12,0 & 81,9 & 0,010 & 0,44 & 0,005 & 0,35 \\
\hline
\end{tabular}

Verifica-se que a rota PCM apresenta uma recuperação mássica global superior mostrando-se uma rota potencial para melhor aproveitamento do minério.

$\mathrm{Na}$ segunda fase duas rotas foram estudadas, para minérios cuja liberação ocorre em malhas inferiores a $0,105 \mathrm{~mm}$. Assim, foram comparadas as rotas PCM com a MFMF para um minério cuja liberação é $0,045 \mathrm{~mm}$. A tabela a seguir apresenta os resultados dos testes, comprovando o desempenho superior da rota PCM.

Tabela 2 - Resultados $2^{\circ}$ Fase - Rota PCM versus MFMF

\begin{tabular}{|c|c|c|c|c|c|c|c|}
\hline \multicolumn{8}{|c|}{ Rota PCM } \\
\hline Fluxo & $\begin{array}{c}\% \\
\text { Massa } \\
\text { BM }\end{array}$ & $\begin{array}{c}\% \mathrm{Fe} \\
\mathrm{BM}\end{array}$ & $\begin{array}{c}\% \mathrm{SiO}_{2} \\
\mathrm{BM}\end{array}$ & $\begin{array}{l}\% \mathrm{P} \\
\text { BM }\end{array}$ & $\begin{array}{c}\% \mathrm{Al}_{2} \mathrm{O}_{3} \\
\mathrm{BM}\end{array}$ & $\begin{array}{l}\% \text { Mn } \\
\text { BM }\end{array}$ & $\begin{array}{c}\text { \% PPC } \\
\text { BM }\end{array}$ \\
\hline ROM & 100,0 & 42,5 & 36,2 & 0,037 & 0,73 & 0,148 & 1,20 \\
\hline Concentrado Concentração Magnética & 73,5 & 50,6 & 24,6 & 0,032 & 0,48 & 0,119 & 1,23 \\
\hline Rejeito Concentração Magnética ROM & 24,5 & 18,0 & 71,3 & 0,050 & 1,49 & 0,246 & 1,14 \\
\hline Pellet Feed & 51,9 & 66,9 & 1,2 & 0,044 & 0,38 & 0,176 & 1,51 \\
\hline Rejeito Concentração Magnética Flotação & 21,6 & 11,6 & 80,8 & 0,021 & 0,73 & 0,061 & 0,55 \\
\hline Rejeito Total & 48,1 & 16,3 & 74,0 & 0,037 & 1,10 & 0,153 & 0,87 \\
\hline \multicolumn{8}{|l|}{ Rota MFMF } \\
\hline Fluxo & $\begin{array}{c}\% \\
\text { Massa } \\
\text { BM }\end{array}$ & $\begin{array}{c}\% \mathrm{Fe} \\
\mathrm{BM}\end{array}$ & $\begin{array}{c}\% \mathrm{SiO}_{2} \\
\text { BM }\end{array}$ & $\begin{array}{l}\% \mathrm{P} \\
\text { BM }\end{array}$ & $\begin{array}{c}\% \mathrm{Al}_{2} \mathrm{O}_{3} \\
\mathrm{BM}\end{array}$ & $\begin{array}{c}\% \text { Mn } \\
\text { BM }\end{array}$ & $\begin{array}{c}\text { \% PPC } \\
\text { BM }\end{array}$ \\
\hline ROM & 100,0 & 42,8 & 36,2 & 0,038 & 0,76 & 0,128 & 1,30 \\
\hline Lamas & 11,6 & 47,0 & 25,5 & 0,095 & 2,83 & 0,268 & 3,50 \\
\hline Pellet Feed & 51,0 & 67,6 & 1,0 & 0,049 & 0,34 & 0,192 & 1,59 \\
\hline Rejeito Concentração Magnética Flotação & 35,3 & 5,3 & 90,7 & 0,003 & 0,70 & 0,029 & 0,17 \\
\hline Rejeito Total & 49,0 & 16,9 & 72,8 & 0,026 & 1,20 & 0,088 & 0,99 \\
\hline
\end{tabular}

$\mathrm{Na}$ terceira fase o foco foi desenvolver uma rota para minérios com liberação em malhas mais grossas e diante dos resultados da segunda fase elaborou-se uma simulação no software Usimpac para a rota MDMF versus a rota PCM e o pré-

* Contribuição técnica ao 44 Seminário de Redução de Minério de Ferro e Matérias-primas, $15^{\circ}$ Simpósio Brasileiro de Minério de Ferro e 2 Simpósio Brasileiro de Aglomeração de Minério de Ferro, 15 a 18 de setembro de 2014, Belo Horizonte, MG, Brasil. 
dimensionamento dos equipamentos possibilitando estimar o CAPEX para cada uma. A rota MDMF apresentou CAPEX 6,5\% inferior a rota PCM. A tabela a seguir apresenta os resultados da simulação.

Tabela 3 - Resultados 3ํFase - Rota MDMF versus PCM

MDMF

\begin{tabular}{l|c|c|c}
\multicolumn{1}{c}{ Fluxo } & $\begin{array}{c}\text { Massa } \\
\text { (\%) }\end{array}$ & $\% \mathrm{Fe}$ & $\% \mathrm{SiO}_{2}$ \\
\hline Alimentação Usina & 100,0 & 41,3 & 38,3 \\
\hline Lamas & 9,8 & 39,9 & 40,4 \\
\hline Rejeito & 42,3 & 12,0 & 80,1 \\
\hline Pellet Feed & $\mathbf{4 7 , 9}$ & 67,4 & $\mathbf{1 , 0}$ \\
\hline PCM & 100,0 & 41,3 & 38,3 \\
\hline Alimentação Usina & 49,6 & 14,6 & 53,6 \\
\hline Rejeito & 50,4 & $\mathbf{6 7 , 4}$ & $\mathbf{1 , 0}$ \\
\hline Pellet Feed &
\end{tabular}

\section{CONCLUSÃO}

A busca de novas rotas motivadas pela necessidade de maximização da recuperação mássica e redução do CAPEX tem promovido ganhos em novos projetos tanto de melhor aproveitamento do minério quanto do ponto de vista econômico.

Neste sentido, a rota de moagem e classificação com P80 próximo a $0,150 \mathrm{~mm}$, seguida de deslamagem, moagem na malha de liberação do underflow e flotação, denominada MDMF, apresentou maior atratividade, devido ao menor CAPEX e menor intensidade de capital ( $\bigcup \$ /$ tonelada produzida).

O estudo continua em andamento e testes piloto serão necessários para confirmar os resultados obtidos na simulação. Além disso, a moagem em malhas mais grossas para ambas as rotas (MDMF e PCM) serão investigadas de modo a reduzir ainda mais a geração de ultrafinos.

\section{BIBLIOGRAFIA}

1 Figueira HVO, Almeida SLM, Luz AB. Tratamento de minérios, 4a revisada, p.113-193, 2004.

2 Rowland CA. Grinding calculations related to the aplication of large rod and ball mills. Allis Chalmers Publications, 1986.

3 Rivera LG. Media Type Effect on Grinding Efficiency. Comminution 2012, Cape Town, April 2012.

4 Wills BA. Mineral Processing Technology. An introduction to the practial aspects of ore treatment and mineral recovery. 6th edition, p. 142-176, 1997.

* Contribuição técnica ao 44 Seminário de Redução de Minério de Ferro e Matérias-primas, 15은 Simpósio Brasileiro de Minério de Ferro e 2ํ Simpósio Brasileiro de Aglomeração de Minério de Ferro, 15 a 18 de setembro de 2014, Belo Horizonte, MG, Brasil. 